\title{
Proceedings of the Association of British Neurologists and the Society of British Neurological Surgeons, Bristol, 4-6 April 1990
}

TOTAL OUTPATIENT SURGICAL MANAGEMENT OF LUMBAR DISC PROLAPSE

HB Griffith, C Marks, AB Jamjoom. Frenchay Hospital, Bristol

Since 1985,50 patients have been treated by microdiscectomy as an outpatient (daycase) procedure. Over the same period we have developed a technique for initial evaluation of the patient utilising the general practitioners' referral findings on examination, together with a history taken over the telephone by the surgeon. In an area with a high standard of general practice it has proved possible to add the history taken by telephone to the general practitioner's findings on examination in order to arrange an outpatient visit for computerised tomography scan. At this same visit the patient's neurological and movement limitation signs are evaluated and a decision made about surgery. This is then carried out as an outpatient procedure. We have followed up the 50 patients by interview and by questionnaire paying attention to the opinions about the effectiveness of surgery $(95 \%$ cured or greatly improved), their tolerance of the surgical procedure as an outpatient, their need for post operative analgesics, and their subsequent mobilisation at home without the need for medical, nursing, or physiotherapy intervention. The vast majority of patients accept the procedure well and would do so again were the need to arise.

THE PERFORMANCE OF SURGIBONE GRAFTS FOLLOWING ANTERIOR CERVICAL DECOMPRESSION

GS Cruickshank, RA Johnston, T Russell, K Grossart. Southern General Hospital, Glasgow and Western General Hospital, Edinburgh

Anterior cervical decompression and fusion surgery for degenerative disease is associated with problems in the behaviour of autologous bone grafts. These include collapse, nonunion, extrusion and altered alignment, in addition to significant donor site complications.

As part of a clinical study, the early performance of a sterilised pretested bovine graf material (Surgibone) has been assessed radiographically.

One hundred and fifty four patients had anterior cervical decompression at one or two levels, by the Cloward technique. One hundred and eighty Surgibone grafts were positioned and assessed by cervical X-ray. This was compared with $\mathrm{X}$-rays taken at three and/or six months and/or one year following surgery.

The performance of the 180 grafts was assessed, by measuring any loss of height in the vertebral bodies bridged by the graft, compared with the fixed height of a single adjacent vertebral body: height loss (rate
$7 \%$ ), graft extrusion (rate $4 \%$ ), vertebral body displacement and alignment change (rate $10 \%$ ). In addition the graft/body interface was qualitatively assessed for signs of bony reaction or fusion: partial (rate $20 \%$ ), complete (rate $55 \%$ ) rates at three months.

The use of prosthetic Surgibone graft in the Cloward procedure allows a more rapid operation without the attendant morbidity of the donor site. The radiological performance of the Surgibone graft compares favourably with that reported for the autologous graft over the period studied.

MORBIDITY AFTER ANTERIOR CERVICAL DECOMPRESSION AND FUSION: TRAIL OF SURGIBONE COMPARED TO AUTOLOGOUS BONE J Rawlinson. Frenchay Hospital, Bristol

In a survey of 89 patients questioned after Cloward's procedure, $73 \%$ suffered more pain from the donor site wound than the neck. This delayed mobilisation in $45 \%$ of patients, and $31 \%$ felt that they could have gone home sooner had it not been for the iliac crest discomfort. In an effort to avoid this morbidity, we set up a trial using Surgibone as an alternative graft material to autologous bone. Surgibone is mature bovine bone wth greater strength than kiel bone.

Eighty nine patients entered the trial. Forty nine were grafted with Surgibone and 40 were grafted with autologous bone. The groups were matched for age and sex. The indications for surgery and the levels decompressed were comparable in both groups. The patients were followed up clinically and radiologically.

The average length of stay in hospital was 5.3 days in the Surgibone group and 5.1 days in the autologous bone group. In the Surgibone group fewer patients were discharged, and there was a significantly higher number of patients who developed new mechanical neck pain after operation.

There was radiological evidence of bony union in more patients in the autologous bone group than in the Surgibone group. The majority of patients with new mechanical neck pain had non-fusion and evidence of movement. This intravertebral movement was more common in those patients with Surgibone who had neck pain after the operation than those without.

Four patients with Surgibone were reexplored: for incomplete decompression (at 11 weeks), wound infection (at 34 weeks) and non-union with mechanical neck pain (at 29 and 46 weeks). Histological examination showed little osteoid being laid down on the Surgibone trabeculae and a florid foreign body giant cell reaction.

Surgibone was not as good as autologous bone for achieving bony union in this study.
POST-OPERATIVE DISC SPACE INFECTION GFG Findlay. Walton Hospital, Liverpool

Post-operative discitis was first described by Turnbull in 1953. The reported incidence varies from $0.2 \%$ to $2 \cdot 8 \%$, with little doubt that heightened clinical awareness leads to an increased diagnostic rate. Whilst diagnosis in established cases is relatively easy to confirm, early diagnosis remains largely clinical.

In over 500 cases of either open or microscopic discectomy, post-operative disc space infection has been diagnosed in nine cases -an incidence of approximately $1.8 \%$. Recently, several experimental and clinical studies have made a strong case for the use of prophylactic antibiotics prior to any procedure which violates the disc.

AUTOMATED PERCUTANEOUS DISCECTOMY, EARLY EXPERIENCE

IJA Robertson, RW Gullan, JR Bartlett. Brook General Hospital, Woolwich

rercutaneous discectomy has been attempted in 14 patients following the acquisition of an Automated Percutaneous Nucleotome. There were 11 men and three women, with a mean age of 37 (range 19-52) years. Twelve patients presented with back pain and sciatica, two with back pain and referred leg pain. Mean duration of symptoms was nine months (one month-seven years). All patients had computerised tomography at the affected level, and also to plan the approach. In addition, nine patients underwent radiculography; two had magnetic resonance scans. Two patients had previously undergone back surgery to the affected level. Eight procedures at the L5/S1 level, six at L4/5 were attempted. In two of the fourteen cases it proved impossible to enter the disc space, both at the L5/S1 disc space. Mean aspiration time in the successful cases was 31 (range 21-54) minutes with 3.5 (range $1 \cdot 0-7 \cdot 5$ ) gms of disc material obtained. Five of the twelve patients who underwent a successful procedure required an open discectomy within three weeks, resulting in an early failure rate of $42 \%$.

UNWANTED EFFECTS AFTER STEREOTACTIC RADIOSURGERY FOR CEREBRAL ARTERIOVENOUS MALFORMATIONS

DMC Forster, JC Sutcliffe. Royal Hallamshire Hospital, Sheffield

More than 350 cerebral arteriovenous malformations have been treated by stereotactic radiosurgery in Sheffield since the unit became operational in September 1985. During that period 10 patients have bled after treatment and six have developed neurological deficits which may have been the result of radionecrosis.

To keep matters in perspective it should be 
remembered that these untoward events have occurred in a population of patients in whom about $75 \%$ have had their arteriovenous malformations obliterated two years and over $30 \%$ one year after radiosurgery.

THE IDEAL TREATMENT FOR CEREBRAL ABSCESS? BA Bell, D Uttley, HT Marsh. Atkinson Morley's Hospital, London

Therapeutic endeavours for cranial abscesses are not a new phenomenon; the skull of Prince Rupert of the Rhine Palatinate was trepanned for an abscess in 1667 in the Stone Gallery of the Palace of Whitehall, and the Prince recovered fully to live a further 15 years. The French surgeon Morand successfully drained an intracerebral abscess in 1768, using his finger to break down the capsule, and a century later Sir William Macewen published a series of 19 patients with brain abscess treated surgically, with 18 recoveries; an enviably low surgical mortality.

Walter Dandy favoured aspiration of the abscess through a burr hole as the treatment of choice in 1926, and Vincent strongly advocated total excision of the abscess in 1936. The controversy between surgical excision and aspiration still persists, and computerised tomography (CT) stereotaxic aspiration has now refined the more conservative surgical technique.

Free-hand aspiration is difficult with deeply-seated, small and multilocular abscesses, and CT guided stereotaxic aspiration has been used in all patients presenting at Atkinson Morley's Hospital since a CT stereotaxic frame has been available. Four patients had supratentorial abscesses, and one an infratentorial abscess and subdural empyema in the cerebellopontine angle. Of those abscesses above the tentorium, one was in the basal ganglia, and one deep parietooccipital, and these would have been particularly difficult to treat by conventional surgical means (see table below).

There was no mortality or morbidity following the procedure, and all patients made a good recovery within weeks of surgery. Stereotaxy coupled with antibiotics given intravenously and into the abscess at the time of aspiration offers accurate drainage with minimal interference to the surrounding normal brain, and is arguably the treatment of choice for all abscesses.

SHORT COURSE ANTIMICROBIAL THERAPY OF INTRACRANIAL ABSCESS: A PRELIMINARY REPORT

A Jamjoom, E Brown, H Griffith. Frenchay Hospital, Bristol

It is standard practice for patients with intracranial abscess to receive antibiotics empirically for six weeks or longer. We suspect that the administration of these agents is being prolonged unnecessarily and currently base the decision to stop treatment on the patient's clinical response and on the fall of the serum C-reactive protein to within the normal range. Serial computerised tomography scans are also performed but they do not necessarily influence the management.

To date, nine patients (five with brain abscess and four with subdural empyema) have been treated using these criteria. The choice of antibiotics was determined by the susceptibility of the isolates obtained at surgery. In the case of 6 patients treatment was stopped after 11-16 (mean 13.5) days. Follow up ranged from two months to one year during which time there were no relapses. The remaining three patients whose abscesses were otogenic received more prolonged courses ( 26 days, 30 days and 10 weeks). In these cases the intracranial sepsis appeared to have been eradicated at an early stage but therapy was extended either because of an undiagnosed deep vein thrombosis (one patient) or failure adequately to treat the underlying aural pathology (two patients).

These preliminary results suggest that pacients with intracranial sepsis may be successfully treated with short courses of an.ibiotics thereby significantly reducing their hospital stay. In patients with abscesses which are otogenic it is essential that the predisposing ear disease is eliminated in order to prevent recurrence or relapse.

MAGNETIC RESONANCE IMAGING (MRI) IN THE ASSESSMENT OF CRANIOSYNOSTOSIS

RD Hayward, DN Grant, B Jones. Hospital for Sick Children, London

Craniosynostosis, as it increases in complex$i t y$, is associated with a wide variety of abnormalities, only some of which are directly connected with the bony deformities of the skull and facial skeleton.

While computerised tomography (CT) scanning (particularly with 3-D reconstruction) is of obvious importance in the planning of reconstructive surgery, MRI provides dramatic evidence of the anatomical malformations that also affect the soft tissues.

Complex cases of craniosynostosis are frequently associated with hydrocephalus and MR scanning has revealed the high incidence of anomalies at the cranio-cervical junction which are responsible for the interference with cerebrospinal fluid flow.

Airway obstruction is usually a significant problem in severe cases and it has recently been recommended that a monobloc advance of the frontal bones and facial skeleton can be performed at an early age in order to overcome breathing difficulties. MR scanning again demonstrates with great clarity the anatomy of the airway obstruction and indicates where craniofacial surgery is likely to be effective.

This presentation from the Cranio-facial Unit at Great Ormond Street demonstrates the value of MRI assessment of a group of

\begin{tabular}{lrlllll}
\hline Patient & Age & Source of infection & Abscess site & Organism & Outcome & Months \\
\hline AD & 61 & Paracolic abscess & Basal ganglia & Coliform & Good recovery & 15 \\
PT & 27 & Cardiac septal defect & Parieto-occipital & Streptococci & Good recovery & 12 \\
RD & 12 & Mastoiditis & CP angle & Anaerobic rod & Good recovery & 7 \\
ST & 8 & Sinusitis & Frontal & Streptococci & Good recovery & 6 \\
IG & 84 & Infected chronic & Frontal & Streptococci & Good recovery & 2 \\
& & subdural & & & & \\
\hline
\end{tabular}

patients who are now receiving increasing neurosurgical attention.

HIGH RESOLUTION ULTRASOUND IN THE ASSESSMENT OF INFANT CEREBRAL TRAUMA BD White, T Jaspan, I Rothwell, JAG Punt. University Hospital, Nottingham

Ultrasound has been used in the investigation of neurological pathology for thirty five years since Leksell demonstrated the ability of echoencephalography to detect midline shift. ' $\mathrm{B}$ '-mode scanning has been used to image neonates' brains since 1980 , but despite a general awareness of its diagnostic potential it has been under-used in the investigation of cerebral trauma. The advent of high resolution ultrasound imaging has greatly enhanced the sensitivity of this technique.

A series of 30 cases is presented, demonstrating the unique advantages of high resolution ultrasound in the evaluation and management of cranial trauma in the infant. We recommend it as a minimally invasive sensitive method of imaging significant pathology which does not require sedation and, importantly, involves no ionising radiation.

SPINAL METASTASES: IS ANTERIOR VERTEBRAL COLLAPSE ALWAYS A CONTRAINDICATION TO LAMINECTOMY?

J Arrotegui, AD Mendelow, R Pickard, PJ Crawford, RP Sengupta, C Barrios. Newcastle General Hospital, Newcastle and Karolinska Hospital, Stockholm

During the period 1982-1988 (Newcastle General Hospital) and 1982-1986 (Karolinska Hospital), we observed 132 patients who had laminectomy for extradural cord compression due to tumour metastases: 39 of these in Newcastle and 93 in Stockholm.

It had recently become generally accepted that laminectomy is hazardous if there is anterior vertebral collapse. Nevertheless, laminectomy remains the most readily available form of biopsy and decompression in many Neurosurgical Units.

The aim of the present study was to audit this practise in relation to vertebral body collapse.

In 132 patients, laminectomy had been performed as the initial therapy followed by other treatment as indicated by tumour histology. Only three patients had a fusion procedure.

The cases were divided into two groups according to outcome.

\begin{tabular}{lllll}
\hline & $\begin{array}{l}\text { No anterior } \\
\text { collapse }\end{array}$ & \multicolumn{2}{l}{$\begin{array}{l}\text { Anterior } \\
\text { collapse }\end{array}$} \\
\hline Ambulant & 35 & $(52 \%)$ & 33 & $(51 \%)$ \\
Not ambulant & 32 & $(48 \%)$ & 32 & $(49 \%)$ \\
Total & 67 & & 65 & \\
\hline
\end{tabular}

The presence of vertebral collapse did not appear to have affected outcome adversely in these patients.

We conclude that laminectomy and tumour removal may stlll be indicated in patients with spinal cord compression, despite anterior collapse, particularly if other techniques of biopsy and decompression are not immediately available. We suggest that where alternative techniques result in delayed diagnosis and decompression, laminectomy may still have a role to play. Prospecive randomised studies would be required to resolve this issue. 
PREDICTION OF DELAYED ISCHAEMIC DEFICIT IN SUBARACHNOID HAEMORRHAGE DG Grosset, I Bone, R Bullock, J Straiton, $M$ du Trevou, D Wyper. Institute of Neurological Sciences, Glasgow

Serial transcranial Doppler (TCD) was performed in 33 selected cases of subarachnoid haemorrhage. Delayed ischaemic deficit (DID) caused permanent deficit in 10 (Group 1) while the remainder made a full recovery (Group 2). The highest achieved mean middle cerebral artery blood flow velocities (FVs) were higher in Group $1(211+/-19 \mathrm{~cm} / \mathrm{sec}$, mean $+1-\mathrm{SE})$ than in Group $2(158+1-$ $9 \cdot 5 ; p=0.03)$, but this variable alone did not usefully predict DID in individual cases. Rapidly rising FVs, often spreading from the site of aneurysm rupture, were more predictive of DID. Nine patients with this pattern had single photon emission computed tomography (SPECT) HM-PAO cerebral blood flow mapping, to compare regional CBF to TCD changes. SPECT was abnormal in eight of nine cases, with hypoperfusion in areas supplied by vessels wth raised FVs in seven cases, and hyperperfusion in one case. Five of the eight patients developed deficit corresponding to TCD changes, and in three of these increased FVs preceded the first clinical signs This relationship between rapidly rising FVs and regional flow reduction on SPECT has not previously been shown. We conclude that TCD can identify vasospasm associated with regional perfusion changes, and in some cases DID can be predicted.

POST-TRAUMATIC CEREBROSPINAL FLUID FISTULAE, THE RISK OF MENINGITIS AND THE CASE FOR SURGICAL REPAIR

M El Jamel, PM Foy. Walton Hospital, Liverpool

The management of acute post-traumatic cerebrospinal fluid (CSF) fistulae is still a matter of debate and a more conservative approach has recently been gaining favour. In order to see if this is justifiable a retrospective analysis of 160 cases of CSF leakage following trauma has been carried out.

The natural history of the CSF leakage and subsequent meningitis has been studied in every patient before and after surgical repair. The mean follow up was 6.5 (range 0.5 to 35 ) years. The overall incidence of meningitis was $33 \%(53 / 160)$. Forty nine patients developed meningitis prior to surgical repair $(30.6 \%)$; the meningitis was recurrent in $30 \cdot 6 \%(15 / 49)$ and was fatal in two $(4 \cdot 1 \%)$. The cumulative risk of meningitis at 10 years follow up was more than $85 \%$, six patients $(4 \%)$ developed meningitis following surgical repair (four had no pre-operative meningitis). The operative mortality was $1.3 \%(2 / 151)$, as was the negative exploration rate. 25/149 $(16.5 \%)$ developed recurrent CSF leakage following the first repair and $9 \%$ developed focal neurological deficits as a result of the operation. Before surgical repair was undertaken, the risk of meningitis was reduced by about $50 \%$ by the use of prophylactic Penicillin and Sulphadimidine. Meningitis occurred in $65 \%$ with no antibiotic prophylaxis and occurred in only $33 \%$ with prophylactic antibiotics.

Surgical repair is the only long term prophylaxis against the development of meningitis following CSF leakage.

\begin{tabular}{|c|c|c|c|c|c|c|}
\hline & & \multirow[b]{2}{*}{$n$} & & \multicolumn{2}{|l|}{ Rebleeds } & \multirow[b]{2}{*}{$p$} \\
\hline & & & & Observed & Expected & \\
\hline \multirow[t]{3}{*}{$\begin{array}{l}\text { Early ( } 0-6 \text { months) } \\
\text { Late (> } 6 \text { months) }\end{array}$} & & $\begin{array}{l}17 \\
15\end{array}$ & & $\begin{array}{l}1 \\
1\end{array}$ & $\begin{array}{l}6 \\
5\end{array}$ & $\begin{array}{l}\text { ns } \\
\text { ns }\end{array}$ \\
\hline & \multirow[b]{2}{*}{$n$} & & \multicolumn{2}{|l|}{ Rebleeds } & & \\
\hline & & & Observed & Expected & $\chi_{y}^{2}$ & $p$ \\
\hline $\begin{array}{l}\text { Early (0-6 months) } \\
\text { Late ( }>6 \text { months })\end{array}$ & $\begin{array}{l}207 \\
147\end{array}$ & & $\begin{array}{l}8 \\
4\end{array}$ & $\begin{array}{l}66 \\
19\end{array}$ & $\begin{array}{r}110 \\
23\end{array}$ & $\begin{array}{l}<0.001 \\
<0.001\end{array}$ \\
\hline
\end{tabular}

REBLEEDING FROM ANTERIOR COMMUNICATING ANEURYSMS AFTER ANTERIOR CEREBRAL

ARTERY LIGATION

WAS Taylor, JD Miller, NV Todd. Southern General Hospital, Glasgow

The long-term risk of rebleeding from ruptured anterior communicating artery (AComA) aneurysms treated by anterior cerebral artery (ACA) ligation was established for 17 patients followed-up for 15-18 years (see table above).

"Expected" numbers of rebleeds were derived from the natural history of untreated, ruptured aneurysms for the period of follow up and calculated from the day of surgery. Although no statistically significant benefit could be identified, the absolute numbers of rebleeds were reduced. With data from six other series it appears that ACA ligation does offer protection from rebleeding. The early rebleeding rate ( $0-6$ months) is $3.9 \%$ and the long-term rebleeding rate is $<1 \%$ per year following ACA ligation (see table above).

Three operations that leave the aneurysm sac patent have been used to treat ruptured aneurysms. These are carotid or ACA ligation and wrapping. They all have a long-term risk of rebleeding of at least $1-2 \%$ per year. Although this is lower than the 3\% risk of rebleeding from untreated ruptured aneurysms it is high when compared with the essentially zero risk of rebleeding after aneurysm clipping. The long-term risk of rebleeding after these operations is also greater than that from incidental aneurysms for which we commonly offer operation. Should patients with ACA (or carotid) ligation be recalled and offered aneurysm clipping?

THE USE AND ABUSE OF EXPERT SYSTEMS BG Mathew, DG Bounds. University Hospital of Wales, Cardiff and Royal Signals and Radar Establishment, Malvern

In the medical context the term "Expert" system is very misleading. A computer cannot give an expert opinion as it is not able to see or examine a patient. The process of diagnosis relies on many visual and other non-verbal clues, an art in which the clinician expert is highly trained. It has also proved extremely difficult to define and model the problem solving logic adopted by clinicians. There is however a place for computerised decisionaids which do not pretend to have expert capability but rather are designed to enhance the skills of the expert clinician.

We have used sophisticated pattern matching software, including "Neural Networks", in the clinical application areas of low back disorders, stroke and acute abdominal pain.
These systems are intended as decision-aids rather than "Expert systems". In each area we have demonstrated a potential for improved patient management particularly wth respect to screening and outcome prediction.

Our experience has led us to conclude that computer based decision-aids do have a defined but limited place in clinical medicine. The technology needs to be simple, cheap and user-friendly and the clinical application areas are limited to those most useful to the busy clinician.

THE IMPACT OF A NEW IMAGE TRANSFER

SYSTEM

T Lee, J Latham, P Anslow, PJ Teddy. Radcliffe Infirmary, Oxford

With the increasing availability of computerised tomography (CT) scanners to district general hospitals and recent advances in electronic and computer technology, a new image-transfer system, "Image Link", has been invented in Oxford and used to link up via ordinary telephone lines the CT scanners in the regional hospitals to our neurosurgical centre. In contrast to the older systems of transferring images, "Image Link" is compatible with different makes of CT scanners, does not require any camera at the transmitting end, takes only about 10 minutes to transfer a complete series of scans and above all, maintains the resolution and clarity of the original pictures. It costs about seven thousand pounds to purchase and install the system and the running cost is that of the telephone calls. We analysed its use in a hundred patients referred to our unit, the majority beng emergency and head injury patients. It has resulted in halting the unnecessary transfer of some very sick or "hopeless" patients as well as early scanning, and urgent transfer of some patients with surgically treatable lesions. "Image Link" has advantages for better patient management, cost-saving and the disadvantage of possible delay of transfer. With the present bed shortage in most neurosurgical centres in the country, and much emphasis on costeffectiveness and ambulance difficulty, it has proved valuable in the management of neurosurgical emergencies.

THE INCIDENCE OF EPILEPTIC SEIZURES AFTER A FIRST EVER STROKE

JPS Burn, PAG Sandercock, J Bamford, MS Dennis and CP Warlow. University of Oxford, Oxford and University of Edinburgh, Edinburgh

Six hundred and seventy five patients with a 
first ever stroke were followed up prospectively from two to six years to detect subsequent seizures. Nineteen $(2 \cdot 8 \%)$ cases had suffered a seizure before the stroke, seven of which were incident within the previous year. Fourteen other $(2.1 \%)$ cases suffered "onset seizures" within 24 hours or during evolution of the stroke. Fifty three cases suffered poststroke seizures of whom $48(7 \cdot 1 \%)$ suffered a first seizure which was not due to a terminal illness or angiographic examination; four had also had "onset seizures". These were, in general, infrequent and easy to control being recurrent in only $25(4 \%)$ cases. Ten patients had their first post stroke seizure more than two years after the stroke; one had also suffered an onset seizure, two had just stopped prophylactic phenytoin and five had suffered a more recent stroke recurrence.

Actuarial analysis estimated that at a year the cumulative risk of having a post-stroke seizure was $4 \cdot 1 \%$ after Cerebral Infarction (CI), $18.2 \%$ after Primary Intracerebral Haemorrhage (PICH), $27.8 \%$ after Subarachnoid Haemorrhage (SAH), and 5.5\% (95\% CI 3.38\%-7.62\%) overall (Logrank test Odds Ratio PICH/SAH vs CI: $10 \cdot 19$ 95\% CI 3.73-27.85). After CI only patients presenting with a "Total Anterior Circulation Syndrome" had a substantial risk $(19.6 \% 95 \%$ CI $5.4-33.8 \%)$ of developing post-stroke seizures within a year of the stroke.

FUNCTIONAL SPECIALISATION OF HUMAN PRESTRIATE CORTEX AS DETERMINED BY POSITRON EMISSION TOMOGRAPHIC STUDIES CJ Lueck,` S Zeki, K Friston, C Kennard, RSJ Frackowiak. The London Hospital, University College and MRC Cyclotron Unit, London

Clinicians have debated the way in which the occipital cortex is organised for over 100 years. The prevailing concept initially was that all visual submodalities (for example, form, colour, motion) were analysed in the primary visual cortex, without regional functional specialisation of the prestriate areas. Several early reports of cases of cerebral achromatopsia due to cerebral vascular lesions suggested that colour might be analysed in the region of the lingual and fusiform gyri. Unfortunately these reports were dismissed, and it was not until the 1970 's that the question of prestriate functional specialisation in man was reopened following findings in non-human primates. However, recent human work has still been based on studies of pathological lesions.

To overcome the objections inherent in this methodology, six normal human subjects were analysed using positron emission tomography (PET). Comparison of scans obtained while subjects viewed displays of either grey versus multi-coloured rectangles, or static versus moving random dots, allowed the demonstration of one area selectively involved in the analysis of colour, and of another, separate, area involved in the analysis of motion. The former was located in the lingual and fusiform gyri, the latter at the posterior end of the middle temporal gyrus.

PET scanning has thus provided clear demonstration of functional specialisation of normal human prestriate cortex

^Awarded the Charles Symonds Prize.

DIASCHISIS, PURE MOTOR STROKE AND SPECT JV Bowler, JPH Wade, BE Jones, DC Costa. Charing Cross Hospital, London
Ischaemic strokes produce immediate focal deficits of cerebral blood flow (CBF) where computerised tomography subsequently shows changes. There are often other regions which show relative reductions in CBF which are thought to be due to transynaptic deactivation (diaschisis).

It has been suggested that recovery from diaschisis is important in recovery from stroke but there is scant evidence for this.

We have studied six consecutive cases of the pure motor stroke due to supratentorial lacunar infarction with serial high resolution SPECT (single photon emission tomography) using a NOVO 810 multidetector dedicated head tomograph, scans usually being done as soon as possible, one week and three months post ictus. The tracer used was ${ }^{99} \mathrm{Tc}^{\mathrm{m}}$ HMPAO (hexamethylpropyleneamine oxime). Clinical deficits were scored for motor function only. Diaschisis was defined as regions of decreased HMPAO uptake in excess of $5 \%$ when compared with the opposite side excluding the site of the infarct. SPECT showed the lesion in four of six first scans. Diaschisis was most often evident in the overlying cortex (eight of 17 scans) but also in the thalamus (five of 17), lentiform nucleus (six of 17), caudate (two of 17) and contralateral cerebellum in two of 15 adequate scans. There was no correlation between the stroke severity score and the extent of diaschisis as assessed by number of involved sites and no significant relation between recovery of stroke score and recovery of diaschisis.

We have been unable to define any role for diaschisis in the production of clinical deficit or in the recovery from pure motor stroke and doubt its importance generally.

INTRACELLULAR CALCIUM AND

OLIGODENDROCYTE INJURY

NJ Scolding, BP Morgan, S Frith, AK Campbell, DAS Compston. Addenbrooke's Hospital, Cambridge and University of Wales, College of Medicine, Cardiff

Mechanisms of myelin damage have been indirectly investigated by studying immunological injury of cultured rat oligodendrocytes. Oligondendrocytes are uniquely sensitive among glia to attack by homologous complement. High concentrations of complement result in cell depth, but during sublethal injury, binding of the terminal membrane attack complex (MAC) of complement triggers a transient rise in intracellular calcium, and oligodendrocytes recover by vesicular removal of the MAC from their surface. Here it is shown that the calcium transient itself triggers vesiculation, acting as a second messenger.

Oligodendrocytes are also shown to be especially sensitive to attack by $\mathrm{T}$-lymphocyte derived perforin, which has functional and structural similarities to complement; it also induces both a calcium transient and vesiculation. Vesiculation can also be stimulated by the calcium ionophore A23187.

Complement-induced calcium-dependent vesicular repair mechanisms have been demonstrated in other cell types. Calcium has a paradoxical role in cell injury in that it is implicated in mediating both cell damage and repair. The sensitivity of oligodendrocytes to injury by calcium-stimulating agents may be a consequence of the unique calcium-dependent metabolic properties of these cells.
XENOBIOTIC METABOLISM IN ALZHEIMER'S

DISEASE

AC Williams, S Sturnam, T Heathfield, G Steventon, $R$ Waring. University of Birmingham, Birmingham

Late onset neurodegenerative diseases could be caused by accumulative damage by chemicals. A relative inability to handle such compounds safely might therefore be a risk factor. $2.5 \%$ of the normal population fail to produce sulphoxides when challenged with S-carboxymethyl-L-cysteine compared with $60 \%$ of patients with a clinical diagnosis of Alzheimer's. The enzyme involved (cystein dioxygenase).converts cysteine to sulphate; cystein:sulphate ratios in Alzheimer's were 477 compared with 96 in controls supporting this concept. Another sulphur metabolising pathway is also defective as when red cel levels of thiolmethyltransferase was assessed levels in Alzheimer's were $291+182$ compared with control values of $947 \pm 409$. Al results significant $p<0.01$. In contrast patients with Alzheimer's have a normal capacity to $\mathrm{N}$-acetylate and $\mathrm{C}$-oxidise xenobiotics.

MICROVASCULAR DECOMPRESSION FOR HEMIFACIAL SPASM AND TRIGEMINAL NEURALGIA: RESULTS IN 4000 CASES

T Fukushima. Mitsui Memorial Hospital, Tokyo

In recent years, suboccipital microvascular decompression has been popularized in neurosurgery, despite some investigators who doubt the neuro-vascular compression mechanism

Ten years experience of microvascular decompression (MVD) comprising 2381 cases of hemifacial spasm (HFS) and 1620 cases of trigeminal neuralgia (TN) is presented.

Male-female ratio was $1: 2 \cdot 2$ for HFS and 1:1.7 for TN. Seven patients had bilateral HFS, 33 cases had bilateral TN and 16 had HFS plus TN. The ages ranged from 17 to 86 (mean 51) years for HFS and from 17 to 95 (mean 62) years for TN. HFS was seen slightly more on the left side and TN more frequently on the right side.

The surgical procedure takes about one hour, is performed via a $1.5 \mathrm{~cm}$ retromastoid craniectomy and consists of identification and transposition of compressing arteries using Teflon tapes.

In HFS, there were 2363 arterial compressions $(99.2 \%)$. One vein, four aneurysms, three arteriovenous malformations and 10 benign tumours $(0.75 \%)$. No explorations were negative.

In TN there were 1377 arterial compressions (85\%), 153 tumours (9.4\%), 11 AVMs $(0.68 \%)$ and 25 veins $(1.8 \%) .54$ patients had negative explorations.

In 10 year follow up the HFS cure rate was $97 \%$ and in TN also $97 \%$. Mortality was $0.13 \%$ (four medical causes, one operative) and morbidity $0.2 \%$ (ataxia, facial palsy, dysaesthesia). Deafness occurred in $1.6 \%$ of HFS and $0.3 \%$ of TN.

The neurovascular compression theory is correct and MVD is one of the safest and most rewarding procedures in neurosurgery.

DOUBLE BLIND STUDY OF BOTULINUM TOXIN A IN TORTICOLLIS

AP Moore, LD Blumhardt. Walton Hospital, Liverpool 
Nineteen patients with torticollis were treated with Botulinum toxin ' $A$ ' (Botox) in a double-blind placebo controlled crossover study. At each visit the clinically most active muscles were injected using one or two injection sites per muscle. Ten patients received Botox $12.5 \mathrm{ng}(500 \mathrm{mu})$ in weeks 0 and 2 and placebo in weeks 12 and 14. Nine patients received placebo first. Using a previously validated scale, responses were assessed "blind" (a) in the clinic and (b) by two observers rating standardised videotapes recorded in weeks $0,4,12$ and 16 . The average of the three scores from each visit was used and objective change was defined as $>30 \%$ alteration in severity. Patients also rated their own responses.

Objective improvement occurred in 12 $(63 \%)$ following Botox and in $4(21 \%)$ after placebo $\left(\chi^{2}\right.$ with Yates correction $=5 \cdot 29, p<$ $0.05)$. Sixteen $(84 \%)$ reported subjective improvement after Botox and $3(16 \%)$ after placebo $\left(\chi^{2}\right.$ with Yates correction $=15 \cdot 15, p$ $<0.01$ ). Two patients deteriorated objectively after placebo, one probably because of declining Botox activity. None deteriorated after Botox. Six (32\%) noted mild dysphagia after Botox and in a seventh pre-existent dysphagia persisted throughout.

This study demonstrates objective improvement with Botox which has previously been hard to document. Optimum dosage, injection techniques and timing have yet to be determined.

\section{ASSESSMENT OF TREATMENT OUTCOME} FOLLOWING RADIOFREQUENCY THERMOCOAGULATION IN 265 PATIENTS WITH TRIGEMINAL NEURALGIA

JM Zakrzewska, DGT Thomas. National Hospital, London

A study was done among 265 patients (mean age 55 years) who had been treated by radiofrequency thermocoagulation (RFT) for trigeminal neuralgia over a 10 year period and followed up for a mean of 43 months (range 1118 months). Twenty patients had either died of unrelated causes or were lost to follow up; one died post-operatively so 244 were circulated with a questionnaire relating to treatment outcome and the Hospital Anxiety and Depression Scale. Of these $179(73 \%)$ were returned. The median time to recurrence was 24 months and at one and five years $63 \%$ and $20 \%$ respectively of patients could be expected to be pain free. There was one death $(0.4 \%)$ and the major morbidity was subjective sensory loss in $135(75 \%)$ of patients of whom $62 \%$ felt it affected the quality of their lives.

In a pre-operative group of 42 patients, $72 \%$ were depressed and anxious whereas post-operatively only $13 \%$ were depressed and $30 \%$ were anxious. One hundred and thirty two $(74 \%)$ said they would undergo the same surgery again if necessary.

RFT provides good pain relief with low morbidity and results in an improved quality of life.

ARE CHILDREN WITH HEAD INJURY SUBJECTED TO IATROGENIC CEREBRAL ISCHAEMIC INJURY? PM Sharples, A McGann, A AynsleyGreen, JA Eyre. Paediatric Research Unit, Newcastle Upon Tyne

Head injury is the most common cause of death and morbidity in children older than one year; establishing its optimal acute management is therefore a major health issue. There are remarkably few data concerning the pathophysiology of traumatic encephalopathy in childhood. Standard therapies are based on the assumption that all children in coma have similar pathophysiology and that cerebral metabolic rates are invariably low. To determine if these assumptions are correct we performed serial measurements of cerebral blood flow (CBF) and cerebral metabolism in 20 children (2-16 years) receiving intensive care following a severe head injury. All had monitoring of arterial and intracranial pressure (ICP). CBF was measured by the Kety Schmidt technique. Cerebral consumption or production of $\mathrm{O}_{2}$, glucose, lactate, pyruvate and ketone bodies was determined from respective arteriojugular venous concentration differences (AJVD) $\times$ CBF. From three to twenty-three serial measurements were performed in each child (157 total). Despite Glasgow coma scores of 3-8, cerebral metabolic rates (CMR) in the first $24-48 \mathrm{hrs}$ were within the normal range. At this time there was uncoupling of CBF and CMR in all but three, with high $\mathrm{AJVOO}_{2}$ and significant cerebral lactate production implying a CBF insufficient to maintain aerobic metabolism. CMR and $\mathrm{AJVDO}_{2}$ tended to fall subsequently. CBF was highly correlated to $\mathrm{PCO}_{2}(\mathrm{p}<0.00001)$. These data show that CMR may be normal in comatose children; routine interventions, such as hyperventilation, which reduce ICP by decreasing CBF may thus be harmful in the immediate post traumatic period.

THALAMOTOMY FOR ALLEVIATION OF LEVODOPA-INDUCED DYSKINESIA

RD Page, MA Sambrook. University of Manchester, Manchester

Levodopa-induced dyskinesia is commonly encountered during the treatment of Parkinson's disease. Clinical and laboratory work has shown that the pathway from the medial segment of the globus pallidus (GPm) to the thalamus is critically involved in dyskinesia such that lesioning the appropriate terminal area in the thalamus could alleviate the disorder. This hypothesis has been tested in a well established animal model.

A group of Macaca fascicularis were rendered parkinsonian by intravenous administration of the neurotoxin 1-methyl-4-phenyl1, 2, 3, 6-tetrahydropyridine (MPTP) and then dosed regularly with levodopa or apomorphine until they were dyskinetic.

To determine the precise thalamic area to be lesioned horseradish peroxidase (HRPWGA) was injected into the GPm of three native macaques and anterograde transport to the area of the thalamus receiving input from the GPm was precisely mapped out. Stereotactic thalamotomy was then carried out in the dyskinetic animals. Levodopa-induced chorea was successfully abolished by lesioning the correct thalamic area whereas control lesions failed to produce a persistent effect.
Dystonia was not relieved by this procedure. Thalamotomy should be re-evaluated as a method of alleviating levodopa-induced dyskinesia, particularly chorea.

PATTERNS OF SEIZURE RECURRENCE IN AN UNSELECTED POPULATION OF PEOPLE WITH NEWLY DIAGNOSED EPILEPSY

YM Hart, JWAS Sander, AL Johnson, SD Shorvon. Institute of Neurology, London and Chalfont Centre for Epilepsy, Bucks

One of the major problems in the study of epileptic seizures is lack of knowledge about the natural history of the untreated condition. In a recent retrospective study of patients attending a neurological department having had two to five seizures, it was found that there was a progressive decrease in the period of time elapsing between successive untreated seizures. If this is so, it would have important implications for the early management of epilepsy.

The National General Practice Study of Epilepsy (NGPSE) is a prospective community-based cohort study of patients with newly diagnosed seizures. Five hundred and ninety six patients of all ages $(52 \%$ male, $48 \%$ female) with confirmed epileptic seizures have been identified and have now been followed for up to five years. Fifty seven per cent of the patients had partial seizures, $34 \%$ generalised, and in $9 \%$ the seizures were unclassifiable. Because patients are identified independently of their hospital referral and the study is prospective, many sources of selection bias are avoided.

We have analysed interseizure intervals between the first and second, and the second and third seizures in cases followed for twelve months or more. Unlike the previous study, we did not find any clear pattern, with many patients showing variation in intervals, and wth great variability across the patient population.

\section{LATE EPILEPSY AFTER ANEURYSM}

B Jennet, I Crandon, M Kay. Southern General Hospital, Glasgow

Epilepsy has been recorded in between 14 $19 \%$ of patients with operated aneurysms. We reported preliminary data to the Society in 1984 and we have now followed 459 cases for at least a year after craniotomy for ruptured supratentorial aneurysms. Epilepsy (after the first week) developed in 14\% (3\%) of single aneurysms, and $17 \%(8 \%)$ of multiple. The epilepsy rate for single aneurysms ranged from $3-25 \%$, according to the aneurysm site and whether there had been any post-operative cortical deficit (temporary or permanent, whether already present before operation or not: (table)). Low-risk cases (3$4 \%$ epilepsy) comprised $38 \%$ of single aneurysms, whilst $35 \%$ were high-risk cases (23-25\% epilepsy). These criteria should make it easier to advise patients about returning to various types of work and resuming driving.

\begin{tabular}{|c|c|c|c|c|c|}
\hline \multirow[b]{3}{*}{$\begin{array}{l}\text { Posterior communicating } \\
\text { Anterior communicating } \\
\text { Middle cerebral } \\
\text { Carotid/ophthalmic }\end{array}$} & \multirow{3}{*}{\begin{tabular}{r}
\multicolumn{1}{l}{$n$} \\
97 \\
142 \\
98 \\
35
\end{tabular}} & \multicolumn{4}{|c|}{$\%$ Late epilepsy ( $95 \%$ confidence interval) } \\
\hline & & All & No deficit & \multicolumn{2}{|c|}{ Deficit } \\
\hline & & $\begin{array}{ll}10 & (6) \\
11 & (5) \\
19 & (8) \\
17 & (12)\end{array}$ & $\begin{array}{rr}4 & (5) \\
4 & (4) \\
14 & (11) \\
7 & (13)\end{array}$ & $\begin{array}{l}18 \\
25 \\
23 \\
25\end{array}$ & $\begin{array}{l}(11) \\
(12) \\
(11) \\
(19)\end{array}$ \\
\hline
\end{tabular}


MRC ANTI-EPILEPTIC DRUG WITHDRAWAL

STUDY

MRC anti-epileptic drug withdrawal group.

Walton Hospital, Liverpool

One thousand and twenty one patients with a history of epilepsy, in remission for two or more years, were entered into a prospective randomized study comparing slow withdrawal of anti-epileptic drugs and continued drug therapy. This group of patients was compared with 776 patients eligible for the study but not entered for a variety of reasons. Randomized patients were more likely to have a history of delayed development and special schooling but were less likely to have a previously attempted anti-epileptic drug withdrawal or to possess a driving licence. They did not differ significantly in other respects.

Eight randomised patients have been excluded from analysis because of ineligibility. In randomized patients the median age of onset was 14 years and the median period seizure free, just over three years. Median duration of epilepsy was five years. $83 \%$ of patients were receiving monotherapy at admission. For those patients with previous EEG reports, $30 \%$ had a generalised paroxysmal abnormality and approximately $40 \%$, some kind of focal abnormality.

Patients have been followed up for between one and five years. Actuarial analysis showed that by three years, $72 \%$ of patients randomised to continued therapy survived seizure free compared to $56 \%$ of patients randomized to slow withdrawal.

\section{A NEW TECHNIQUE OF NONINVASIVE}

INTRAOPERATIVE MOTOR EVOKED POTENTIAL MONITORING

DA Jellinek, DA Jewkes, MW Platt, L Symon. National Hospital, London

We wish to present a new technique of noninvasive intraoperative motor evoked potential monitoring. The technique employs total intravenous anaesthesia with Propofol and partial neuromuscular blockade with atracurium besylate. Motor cortex stimulation is delivered transcranially. Single EMG responses from peripheral muscle are employed to assess function of the motor pathways.

This technique has been employed on 29 patients undergoing neurosurgical procedures at the National Hospital for Nervous Diseases with a success rate of $90 \%$. The technique has been of specific value as an intraoperative monitoring tool in four patients: one anterior communicating artery aneurysm, one spinal neurofibroma, and two spinal arterovenous malformations. On each occasion motor evoked potential monitoring yielded useful diagnostic information.

We consider that this technique will facilitate spinal cord monitoring in neurosurgery, supplementing somatosensory potentials which have been demonstrated to be an unreliable indicator of spinal cord damage.

THE DIAGNOSIS OF NORMAL PRESSURE HYDROCEPHALUS

DJ Price. Leeds General Infirmary, Leeds

Normal Pressure Hydrocephalus (NPH) due to a cerebrospinal fluid (CSF) malabsorption defect of unknown aetiology is easy to treat but difficult to diagnose. Many thousands of our population present with less than a two year history of at least three of the four clinical characteristics of this condition and on computerised tomography examination have hydrocephalus with relative effacement of the arachnoid space near the vertex. Only some however have NPH and the others have multi-infarction dementia, Alzheimer's Disease or just atrophy.

In an initial series of 20 patients with these clinical and radiological features, a measurement of the outflow resistance of CSF by a ventricular infusion technique was followed by a shunt in all of them. Only six wth outflow resistances of above $14 \mathrm{mmHg}$ / $\mathrm{ml} / \mathrm{min}$ improved clinically. Four of the other patients developed complicating chronic subdural haematomas requiring drainage.

In the most recent series of 77 similar patients, only the 41 with resistances of more than $14 \mathrm{mmHg} / \mathrm{ml} / \mathrm{min}$ were treated with a shunt. The probability of a good response for at least a year was directly proportional to that outflow resistance.

In future, a more objective measurement of the ventricular-cortical sulcal ratio by magnetic resonance scanning may well prove more effective than computerised tomography scanning for the initial selection of patients for this invasive measurement of outflow resistance.

THE POSSIBLE NEED FOR INTRA-CRANIAL EEG IN SURGERY FOR TEMPORAL LOBE EPILEPSY GDS Wright, JM Oxbury, P Fenwick, SM Oxbury, CBTA Adams. Radcliffe Infirmary, Oxford

Since 1982, 125 patients with drug-resistant psychomotor epilepsy have been considered for temporal lobe surgery (en bloc excision or amygdalohippocampectomy). The investigation procedures available have been routine, sleep and sphenoidal EEG, neuroradiology and neuropsychology including intra-carotid amytal.

Seventy four patients underwent temporal lobe surgery. Twelve were on the basis of the clinical presentation combined with neuroradiology and neuropsychology findings, and 62 on the basis of these along with EEG data, particularly those from sphenoidal recordings. Three others had non-temporal lobe surgery.

The remaining 48 have been surveyed to determine how many might have been offered surgery if more sophisticated EEG techniques, such as intra-cranial recording, had been available. Nine became adequately controlled. Two were considered to have pseudoseizures. Two declined operation. Eighteen had evidence of bilateral and/or non temporal pathology, derived from neuropsychology and/or neuroradiology, which would have made them unsuitable for surgery. Only in 17 cases, $14 \%$ of the original population, was surgery rejected solely because no single temporal EEG focus could be established with the techniques available even though neuroradiology and neuropsychology findings were consistent with a unilateral temporal lobe abnormality.

We conclude that relatively few patients referred for temporal lobe epilepsy surgery might benefit from intra-cranial EEG recording.
STEREOTACTIC SURGERY IN THE MANAGEMENT OF 200 CONSECUTIVE INTRACRANIAL MASS LESIONS. ANALYSIS OF INDICATIONS, BENEFITS AND OUTCOME

AM Wild, PV Marks, JH Xuereb, JRW Gleave. Addenbrooke's Hospital, Cambridge

Two hundred consecutive stereotactic procedures were performed. These were 151 biopsies and 45 therapeutic procedures. The latter included aspiration of craniopharyngiomata, arachnoid cysts, deep seated abscesses, and the destruction of colloid cysts. Cryogenesis was used to treat an arteriovenous malformation in one case. In four cases the system was used to locate lesions during open surgery. Leksell Stereotactic Systems were used throughout. The ages ranged between 12 months and 83 years.

Overall in $34 \%$ of biopsies the preoperative diagnosis was not confirmed. When the provisional diagnosis was glioma, only $63 \%$ were confirmed; $17 \%$ were found to be benign, and $14 \%$ some other form of malignancy. Biopsy provided tissue on which a histological diagnosis could be made in 147 procedures. In 10 cases the biopsy had to be repeated; in nine of these a positive diagnosis was subsequently made, the tenth showing gliosis on both occasions. Mortality was $1.5 \%$; there was a transient deterioration in $5 \%$ and prolonged deterioration in $1.3 \%$.

These observations suggest that stereotactic surgery is far superior to freehand burr hole biopsy and exploratory craniotomy in the management of these lesions. No radiation therapy should be considered without histological confirmation.

THE PROGNOSIS OF PRIMARY INTRACEREBRAL TUMOURS PRESENTING WITH EPILEPSY: THE OUTCOME OF MEDICAL AND SURGICAL

MANAGEMENT

DF Smith, D Sandeman, PM Foy, MDM Shaw, IR Williams, DW Chadwick. Walton Hospital, Liverpool

There is increasing pressure for all patients presenting with epilepsy to have computerised tomography scanning to exclude the presence of intracranial tumour even though this is a rare cause of epilepsy in most age groups. This policy might in part be justifiable if early surgical intervention could be shown to influence the long-term outcome of primary intracerebral tumours causing epilepsy.

We have retrospectively examined the prognosis in a group of 158 patients whose first symptom of primary intracerebral tumour was epilepsy. One hundred and seven patients had a histologically proven diagnosis. Of these 78 were diagnosed during the initial referral and investigation process, and 29 were diagnosed after a long history $(>2$ years) of drug-resistant epilepsy. In 51 patients the diagnosis was made on clinical and radiological grounds without histological evidence.

Survival is significantly better for this group of patients than 466 patients with other presentations of primary intracerebral tumours.

Primary intracerebral tumours presenting with epilepsy appear relatively benign and the value of early surgical intervention needs to be tested in a prospective study. 
LONG SURVIVAL IN PATIENTS WITH SUPRATENTORIAL MALIGNANT GLIOMAS EXHIBITING NEURONAL DIFFERENTIATION JS Garfield, RO Weller. Wessex Regional Neurological Centre, Southampton

Between 1976 and 1984, in the European Organisation for Research and Treatment of Cancer randomised studies, the best median survival following surgery and radiotherapy, with or without adjuvant therapy, was 20 months. Of the 90 patients from Southampton, $26(29 \%)$ survived longer than 24 months. The average periods from surgery to death or of known survival in these cases were: 71 months (trial 26781), 53 months (26751) and 64 months (26812).

Nine of the 26 long surviving Southampton patients (mean age at surgery 44 years) showed histological and immunocytochemical evidence of neuronal differentiation in the tumour biopsy. Four of these nine patients are still alive (age range $29-47$ years at surgery, survival 45-114 months) and 5/9 are dead (age range 41-58 years at surgery, survival 24-54 months). More than half of the neuronal tumours showed areas of necrosis and capillary endothelial proliferation. Mitoses were present in all tumours.

It appears that histological and immunocytochemical features of neuronal differentiation are favourable prognostic factors which can only be established following surgery and wide tumour sampling. This, with other favourable prognostic factors, and the expected $29 \%$ survival for longer than 24 months, lends weight to an active rather than to a nihilistic approach to patients with a pre-operative diagnosis of a supratentorial malignant glioma.

MAGNETIC RESONANCE SCANNING OF THE CERVICAL SPINE-AN UNEXPECTED PROBLEM SM Marks, $R$ Parameswaran. Middlesbrough General Hospital, Cleveland

Magnetic Resonance Scanning (MRI) has recently become more readily available and it has been suggested that this investigation will replace conventional myelography.

During a period of 14 months cervical myelography was unavailable in our unit and patients requiring this investigation were referred for Magnetic Resonance Scanning to two centres in London. Cervical myelography if required, however, was available at the Newcastle Unit, and patients requiring urgent investigations or who were unfit or unable to travel were submitted directly for cervical myelography. Only patients under the care of the first author were included in the study and they were all personally assessed and operated on by him. Vascular and neoplastic conditions are excluded as only patients with cervical degenerative disease are reported. Those who underwent cervical myelography directly are also excluded. A total of $\mathbf{3 6}$ patients was referred for MRI as the initial definitive investigation. Only one patient could not tolerate the procedure and three patients moved, producing some artifact, however the radiologist was able to submit a report. Fourteen patients including the four above then had a cervical myelogram. The reason was that the MRI scan was not consistent with the neurological assessment and a management decision could not be made. Most of the patients were then shown to have a definitive consistent lesion and underwent appropriate surgery whence the pathology was confirmed. The lesions not revealed by $M R I$ included both cord and root compression. The other 22 patients had consistent MRI scans and most underwent appropriate surgery. The MRI scans were all reported by experienced neuroradiologists who were supplied with full clinical details. The authors reviewed all scans and agreed with the formal reports.

The authors cannot entirely explain why the MRI scan failed to reveal the lesions in $\frac{1}{3}$ of patients examined. We suggest that at present MRI scanning is not the investigation of choice in cervical degenerative disease.

BASILAR INVAGINATION AND CLINICAL SIGNS: IS THERE A CORRELATION?

HA Crockard, HL Harkey, JM Stevens. National Hospitals, London

Attention to the association of clinical features and severe basilar invagination was drawn in the first half of this century. Whether specific neurological features and the anatomical deformity are correlated remains unclear. The availability of CT and MRI has allowed a detailed clinicoanatomical review.

Twenty-nine patients with basilar invagination have been evaluated in this department since 1980 . There were four main groups of craniovertebral anomalies associated with the basilar invagination. Odontoid translocation in i) rheumatoid arthritis-nine patients, ii) os odontoidium anomalies-five patients, iii) Klippel Feil deformities-nine patients and iv) osteogenesis imperfecta-six patients.

In group i) there was marked anterior brain stem compression due to the translocated peg. There were no cranial nerve signs or nystagmus but long tract signs were present. Group ii) were younger, some with differences in limb size, all with long tract signs but no cranial nerve deficit or nystagmus. Those with Klippel Feil deformities (Group iii) were of two types either occipito-atlantal fusions and basilar invagination or more complicated with accessory condyles. Four had additional Arnold Chiari malformations, and in them there were widespread neurological features. The most severe deformities were noted in osteogenesis imperfecta (Group iv) with neuraxial deformities extending up to the mid brain. These cases showed marked cranial nerve deficits, nystagmus and long tract signs.

There was no correlation between neuraxial angulation and the resultant signs, but the circumferential compression and its extent were more important. The cross sectional area of the cord and brain stem was related to the neurological disability.

IDENTIFICATION AND MANAGEMENT OF CRITICALLY-IMPAIRED CEREBRAL PERFUSION IN PATIENTS WITH EXTRACRANIAL VASCULAR DISEASE

RJ Nelson, JC Stanley, J Webster, AHJ Lovick, S Powell, HK Richards, J Roberts, JD Pickard. Southampton General Hospital, Southampton

Symptomatic cerebral ischaemia in patients with extracranial atherosclerosis is usually attributed to thrombo-embolism. Reversible haemodynamic insufficiency is recognised as a cause of cerebral ischaemia but is thought to be rare and difficult to diagnose without sophisticated investigations. We believe there is a group of patients with critically impaired cerebral perfusion who can be identified by their clinical presentation and by noninvasive assessment of their cerebral haemodynamics.

Two hundred and twenty patients referred to a specialist cerebrovascular clinic have been investigated by Duplex scanning of the carotid and vertebral arteries, transcranial Doppler scanning of the circle of Willis with tests of intracranial collateral circulation, ${ }^{133}$ Xenon measurement of regional cerebral blood flow and cerebrovascular reactivity to hypercapnia, computerised tomography scanning and, as necessary, digital subtraction angiography. Seventeen men and one woman (mean age 65) were considered to have critically impaired cerebral perfusion and underwent early carotid endarterectomy with intraoperative carotid shunting and cerebral blood flow monitoring. They were characterised by:

$1 \mathrm{~A}$ history of recurrent transient ischaemic attacks and/or completed minor stroke with good neurological recovery.

2 Non-focal neurological symptoms of dizziness, ataxia and intellectual deterioration.

3 CT scan evidence of multifocal lacunar and/or watershed cerebral infarction often with cortical cerebral atrophy.

4 Bilateral carotid disease: bilateral stenoses $>75 \%$, or occlusion and contralateral stenosis $>50 \%$.

5 Impairment of cerebrovascular reactivity, $<1 \% / \mathrm{mmPaCO}_{2}$, or inverted reactivity

6 Predominantly anterior collateral flow often with turbulence in the anterior communicating artery.

The surgical mortality of $11 \%$ was related to postoperative myocardial infarction. The stroke-free survival at January 1990 was $72 \%$. There were postoperative improvements in both cerebrovascular reactivity and cerebral blood flow velocity. Although these are high-risk patients with a poor prognosis their investigations indicate that they will not tolerate a further reduction in cerebral perfusion and surgery appears justified.

SYMPTOMATIC CAROTID ISCHAEMIC EVENTS: WHAT IS THE SAFEST, LEAST COSTLY AND MOST EFFECTIVE CAROTID ARTERY IMAGING

STRATEGY TO SELECT PATIENTS FOR CAROTID ENDARTERECTOMY?

GJ Hankey, CP Warlow. Western General Hospital, Edinburgh

Two hundred and ninety six patients with transient ischaemic attacks or retinal infarction were evaluated to determine the validity of an ipsilateral carotid bruit in predicting stenosis of the origin of the symptomatic extracranial internal carotid artery (ICA) and the influence that a carotid bruit should make on the clinical decision to proceed with ultrasonographic or angiographic investigation. The prevalence of mild disease (stenosis $\geqslant 25 \%$ ) of the symptomatic ICA stenosis rose to $92 \%$. The prevalence of moderate stenosis of the symptomatic ICA (stenosis $\geqslant 50 \%$ ) was $39 \%$ and if a bruit was heard the probability doubled to $78 \%$. The prevalence of severe ICA disease ( $\geqslant 75 \%$ stenosis) was $22 \%$ and if a bruit was heard the probability was $49 \%$, more than double. Thus, the presence of a bruit was a useful predictor ( $92 \%$ of cases) of mild ICA disease but it had a greater impact in changing the post-test 
probability of more severe degrees of ICA stenosis.

The direct cost of several carotid artery imaging strategies was expressed in terms of the number of pounds and post-angiographic strokes it costs to detect patients with various grades of diameter stenosis. If clinicians aim to detect stenosis of greater than $25 \%$, it is most cost-effective to proceed directly to cerebral angiography in patients with a carotid bruit over the symptomatic carotid bifurcation and to screen only those patients with no bruit by Duplex carotid ultrasound. Those patients in whom Duplex reveals ICA stenosis of greater than $25 \%$ are then referred for cerebral angiography. If clinicians wish only to detect more severe ICA disease (stenosis $\geqslant 50 \%$ ), then the same policy applies unless the local Duplex service is particularly efficient and reliable, in which case it is probably more cost-effective and safer to screen all patients with Duplex irrespective of the findings of cervical auscultation. If clinicians aim to detect ICA stenosis of $75 \%$ or greater then it is clearly most cost-effective to screen all patients with Duplex, whether a bruit is present or not, because this approach will reduce the number of angiograms required, be less expensive, and result in far fewer post-angiographic strokes.

XENON-ENHANCED CT MEASUREMENT OF CEREBRAL BLOOD FLOW IN CEREBROVASCULAR DISEASE

MM Brown, DM Pelz, V Hachinski. St George's Hospital, London and University Hospital, London (Ontario)

Cerebral blood flow (CBF) was measured in patients with cerebrovascular disease using the stable xenon computed tomography (CT) technique on a General Electric 9800 scanner. Regional CBF was calculated from the rate of $C T$ image enhancement resulting from the inhalation of a non-radioactive xenon-oxygen mixture. The resulting cross-sectional axial CT images of regional CBF were found to have excellent anatomical resolution of cortex, deep nuclei and white matter. The technique clearly delineated areas of infarction and luxury perfusion following stroke, and spasm following subarachnoid haemorrhage. A normal range of resting regional CBF in grey and white matter was established in normal elderly volunteers. The measurements were repeated after the injection of acetazolamide to determine vasodilatory reserve. The results were compared with measurements in 10 patients with cerebrovascular disease found to have diffuse periventricular white matter lucency on CT. There was no difference in resting cortical or deep white matter CBF between the groups. After injection of acetazolamide, cortical and white matter CBF increased markedly in the normal group. In contrast, in the patient group cortical flow increased normally but there was no significant increase in white matter flow. The results suggest that haemodynamic ischaemia may be responsible for diffuse white matter lucency in cerebrovascular disease.

WHAT IS THE NATURAL HISTORY OF NEUROSURGICAL DISEASE IN THE ABSENCE OF NEUROSURGICAL INTERVENTION?

JD Pickard, S Bailey, H Sanderson, $M$ Rees, et al. Wessex Neurological Centre, Southampton
As part of our Cost Benefit Analysis of Regional Neurosurgical Care, first reported in September 1987, we have had to define the expected outcome in the absence of neurosurgical intervention. The prognosis for conservatively managed subarachnoid haemorrhage patients has been well-defined but this is not true for many other neurosurgical conditions. Following discussion with B Jennett and M Buxton, the panel approach was used to provide a measure of the range of experienced opinion as to the expected outcome without neurosurgery.

Twenty one United Kingdom consultant neurosurgeons of all ages were invited individually to estimate outcome for 45 diagnoses, distributing percentage probabilities for each of the five possible outcomes on the Glasgow Outcome Scale, modified as necessary for non-lethal disease, at 6 months and after 10-20 years. The spectrum of severity of presentation for each diagnostic category was defined as that to be expected for cases referred to a British Regional Neurosurgical Unit. Seventeen consultant neurosurgeons replied within 24 hours to one year. The mean and median values, and $95 \%$ confidence intervals were calculated. All members of the panel found the exercise demanding but instructive as evidence by their comments and the $95 \%$ confidence limits.

CASE SELECTION OF PATIENTS FOR TEMPORAL LOBE SURGERY: A PROSPECTIVE FOLLOW-UP STUDY

RDC Elwes, M de Silva, CD Binnie, EH Reynolds. King's College Hospital, London

Recently there has been increasing interest in the surgical treatment of epilepsy. The techniques are available in only a few specialised centres. In order to allow for the rational use of scarce and expensive facilities some estimate of the total numbers of patients requiring surgery is needed.

Since 1981 we have followed prospectively all adults and children referred with newly diagnosed and previously untreated epilepsy. Out of a total of 237 patients who have been followed for two or more years $33(14 \%)$ have failed on drug treatment and have chronic intractable epilepsy. Five patients $(2 \%$ of the total) are experiencing at least one complex partial seizure (CPS) a month and have a clear unilateral temporal lobe focus on the interictal EEG. A further 10 patients have CPS but need telemetry with either scalp, foramen ovale or depth electrodes to indicate the site of the focus.

Our experience, based on a preliminary assessment is that at least $2 \%$ of all newly diagnosed epileptic patients may be suitable candidates for anterior temporal lobectomy or amygdalohippocampectomy.

DISTURBANCE OF TASTE AND SMELL AFTER CLOSED HEAD INJURY: ANATOMICAL

RELATIONSHIPS

R Grant, K Weidmann, D Hadley. Western General Hospital, Edinburgh

Abnormalities of taste and smell are well recognised complications of closed head injury (CHI). The relationship between disturbances of these primary senses and anatomical site of injury have not been adequately studied due to technical difficulties in measuring these senses accurately and of localising the site of injury. The introduc- tion of Magnetic Resonance Imaging (MRI) and a simple standardised quantitative electrogustometric technique has now made accurate assessment possible. Thirty patients with $\mathrm{CHI}$ who were admitted to hospital underwent MRI scanning in the acute stage. Assessment of taste and smell were performed five to 18 months after their injury using a standardised electrogustometer and four different aromatic solutions. Thirteen patients had persisting abnormalities of taste and 12 had olfactory obnormalities. Seven patients had abnormalities of both. Eleven of 12 patients with abnormal olfaction had frontal lobe lesions compared with 10 of 18 patients with normal smell ( $p<0.05)$. Ten of the 13 patients with disturbances of taste had brain stem lesions on MRI compared with ive of 17 patients with normal taste $(p<$ 0.01 ) and 13 patients with disturbances had lesions in the temporal lobe compared with only seven patients who did not have taste disturbance $(p<0.05)$. Complete ageusia occurred more frequently with brain stem lesions. Persistent disturbances in taste and smell are common after moderately severe $\mathrm{CHI}$ and may be more common in the early stages. The presence of these abnormalities on clinical examination increases the likelihood of an abnormal MRI scan.

SPONTANEOUS CAROTID-CAVERNOUS FISTULA IN FIBROMUSCULAR DYSPLASIA: CASE REPORT DG Grosset, R Bullock, DM Hadley, E Teasdale. Southern General Hospital, Glasgow

Fibromuscular dysplasia (FMD) is often associated with intracranial aneurysms and haemorrhage. We report the much rarer occurrence of carotid-cavernous fistula, with an assessment incorporating cerebral blood flow studies.

A 29 year old woman had three weeks of constant headache and cervical pain and noted the onset of a bruit in the right side of head. Examination found bruits in the right carotid and fronto-temporal regions.

Magnetic resonance imaging showed an engorged right cavernous sinus and enlarged superior ophthalmic vein. Cerebral angiography showed the "string of beads" appearance of FMD in the right internal carotid (ICA) and right vertebral arteries, and a high flow right carotid-cavernous fistula. During two minutes of compression of the right carotid artery in the neck, right hemisphere blood flow (Xenon and single photon emission computed tomography) fell by $20 \%$ and there was marked slowing of electrencephalographic rhythms. Transcranial Doppler sonography (TCD) showed right middle cerebral artery flow fall to zero after carotid compression, with a return to normal flow velocities at 20 seconds by collateral flow.

Balloon occlusion of the fistula was carried out. It proved necessary also to occlude the right ICA. Collateral flow typical of high ICA occlusion was identified by TCD.

MOYA MOYA DISEASE: CASE REPORT IN

PREGNANCY INCLUDING TRANSCRANIAI

DOPPLER EVALUATION

DG Grosset, P Barlow, I Bone. Southern General Hospital, Glasgow

A 29 year old Caucasian woman at 15 weeks gestation presented with sudden onset of headache, vomiting and photophobia. 
Cerebrospinal fluid examination confirmed the clinical diagnosis of subarachnoid haemorrhage (SAH). Computed tomography showed extensive subarachnoid and intraventricular blood, hydrocephalus and established infarcts in left prefrontal and parietal regions.

Cerebral angiography showed left internal carotid artery (ICA) occlusion at syphon level, with extensive collateral Moya Moya vessels and a right meningeal to left middle cerebral artery collateral supply. Further left hemisphere supply was from the anterior communicating artery and the basilar artery.

The proximal left ICA had an altered Doppler waveform characteristic of distal obstruction. Left middle and anterior cerebral artery flow velocities were low due to the carotid occlusion. Right hemisphere and basilar artery velocities were one and a half times normal. Signals of three times normal velocity represented flow in Moya Moya vessels.

There was no Doppler evidence of vasospasm. The patient was managed conservatively with elective Caeserean section at 38 weeks.

This is the first reported case of subarachnoid haemorrhage in pregnancy due to Moya Moya disease in a non-Japanese patient. Two previous cases originated in Japan, where the disease was described and remains more common.

DOES CHRONIC LOW DOSE EXPOSURE OF SODIUM NITRATE INCREASE GLIOMA FORMATION IN VMSTRAIN MICE?

CH Hawkes, JB Cavanagh, J Darling, B Watkins, DGT Thomas. Institute of Neurology, London

There is evidence that either nitrite or nitrate may be carcinogenic through conversion to nitrosamines. This theory has received support with respect to gastric carcinoma but has not been considered in connection with cerebral glioma. This observation and the existence of slightly elevated Standardised Mortality Ratios for glioma in East Anglia, an area known to have longstanding high nitrate levels in soil and drinking water prompted further investigation. In the VM strain of mice $1 \cdot 6^{\circ}$ onimals spontaneously develop cerebral glioma hence it was thought reasonable to test the hypothesis that chronic exposure to sodium nitrite would elevate their natural risk to such malignancy. Five hundred VM mice of both sexes were used as follows: 1) Control group (200) which drank distilled water 2) Experimental group $a(200)$ which from the time of weaning drank distilled water containing $0.2 \%$ sodium nitrite. Experimental group $b(100)$ were exposed to the same concentration during the whole of pregnancy as well as adult life. All animals were kept until natural death or until severe illness developed when they were sacrificed. The brains and spinal cords were removed, fixed in formalin and stained with haematoxylin and eosin. Two tumours developed in the experimental group. One was a typical intrinsic glioma and the other possibly a secondary deposit in the spinal canal. In the control group one tumour was discovered which had arisen from the base of the brain or pituitary region. Sodium nitrite does not increase the incidence of cerebral glioma in this strain of mice.
COLLATERAL SPROUTING OF SENSORY NERVES IN MAN

C Healy, PM Le Quesne, B Lynn. The Middlesex Hospital, London

Clinical studies have suggested that collateral sprouting of adjacent intact nerves may contribute to the return of cutaneous sensation following nerve injury in man. Animal experiments have shown that nociceptive Ad and $C$ fibres but not large diameter mechanoreceptive fibres may sprout. The process is more active when the nerves producing the sprouts are partially damaged.

A suitable lesion for quantitative study in man is produced when $15-20 \mathrm{~cm}$ of the medial antebrachial cutaneous nerve in the upper arm is removed for use as a nerve graft. This results in a sensory deficit of approximately $20 \times 10 \mathrm{~cm}$ in the upper forearm. The length of nerve resected is considerably in excess of the critical gap for regeneration; the surrounding skin is innervated by undamaged nerves.

Five subjects have been followed from the time of operation for up to 15 weeks. The areas of sensory loss to modality specific suprathreshold stimuli were mapped. The deficit was always largest for warming sensation and smallest for noxious heat. There was no detectable change with time for touch, warming or cooling. Mean width of the deficit decreased by a small amount for sharp pain, from 9.1 (SE 0.66) to 8.2 (SE 0.59 ) cm ( $p<0.005$ for paired test); and for noxious heat, from 7.8 (SE 0.87 ) to $6.6($ SE 0.66$) \mathrm{cm}$ $(\mathrm{p}<0.02)$.

DIFFERENTIAL INVOLVEMENT OF PROXIMAL MUSCLES IN MYASTHENIA

N MacDermott, W Schady. Manchester Royal Infirmary, Manchester

High frequency repetitive nerve stimulation is often used to diagnose $m_{y}$ asthenia. Proximal muscles are more likely to yield abnormal results than hand muscles. The trapezius has been favoured because its stimulation is less painful than that of deltoid or biceps. We have undertaken 42 repetitive stimulation studies in both deltoid and trapezius on 32 adult myasthenics off medication. Each study consisted of a baseline train of eight supramaximal impulses at $2 \mathrm{~Hz}$, two similar trains at one and two minutes after a 15 second maximal voluntary contraction (MVC), a 3 $\mathrm{Hz}$ train at 3 minutes and a $5 \mathrm{HZ}$ train at 4 minutes.

The mean baseline decrement in trapezius and deltoid was $9 \%$ and $24 \%$ respectively ( $p$ $<0.001$ ), while after MVC corresponding values were $12 \%$ and $32 \%(2 \mathrm{~Hz}), 12 \%$ and $34 \%(3 \mathrm{~Hz})$, and $14 \%$ and $32 \%(5 \mathrm{~Hz})$. Decrement was greater in deltoid than trapezius in $79 \%$ of baseline trains. Results were diagnostic in deltoid but normal in trapezius in 13 studies, while the reverse occurred in only one. We conclude that, when testing endplate function, (1) deltoid is more likely to yield conclusive results than trapezius, (2) post-tetanic exhaustion may enhance the decrement, and (3) there is no need to use stimulus frequencies greater than $2 \mathrm{~Hz}$.

SPECIALISED EPILEPSY CLINICS-A

RANDOMISED CONTROLLED TRIAL

JI Morrow, A Richens. University Hospital of Wales, Cardiff

The management of epilepsy in the United Kingdom has been criticised and as a method for improving standards specialised epilepsy clinics have been recommended. The concept, however, remains largely controversial. Specialised epilepsy clinics have been established particularly for severe epilepsy, but can they offer more than existing services?

In order to evaluate the work of a specialised epilepsy unit, patients with epilepsy or suspected epilepsy referred to the University Hospital of Wales were randomised to attend either a standard neurology clinic or the specialised epilepsy unit.

Patients were assessed prior to their initial attendance and reassessed at three, six and 12 months. Two hundred and thirty two patients were included in the study ( 91 males and 41 females, mean age 32 years).

Patients attending the epilepsy unit were seen more often and were less likely to be discharged. They had more EEG's and plasma drug estimations than those attending the neurology clinic.

Measures of seizure frequency demonstrated improvements in both groups over baseline, though these improvements were greater in the Epilepsy Unit patients ( $p<$ 0.05). More patients in the Epilepsy Unit group achieved plasma concentrations within the therapeutic range ( $p<0.001$ ), and fewer reported adverse effects $(p<0.05)$.

MAO-B ACTIVITY IN PLATELETS OF UNTREATED PARKINSON'S DISEASE

G Steventon, AC Williams, S Sturman, $\mathbf{R}$ Waring. University of Birmingham, Birmingham

Inhibition of monoamine oxidase-B (MAO$B$ ) by Selegiline may retard the progression of Parkinson's disease and certainly prevents 1-methyl-4-phenyl-1, 2, 3, 6-tetrahydropyridine toxicity. We have studied this enzyme in untreated patients and compared them with age matched controls.

In this study, Dopamine was used as substrate. Male patients $(n=9)$, produced 224 $\pm 37 \mathrm{nM}$ Dopac/mg platelet protein/hour compared with control values of $451 \pm 63$.

Female patients, $(n=8)$ also showed reduced activity at $235 \pm 19$ compared with control activity of $551 \pm 60$ (p values all $<0.001$ ). A previous study in contrast showed increased enzymic activity using a different substrate (phenylethylamine). In both studies there was very little overlap with the apparently normal range. These results, in combination, suggest that MAO-B in patients with Parkinson's is not dysregulated, but may be structurally different.

PATTERNS OF MOVEMENT IN SPASMODIC TORTICOLLIS

CD Ward, P Critchley. University of Southampton, Southampton

In the treatment of torticollis with botulinum toxin, the injection of ipsilateral splenius capitis (SC) and contralateral sternomastoid (SM) is advocated, based on the assumption that lateral rotation is caused by over-activity of prime movers. We tested this hypothesis by examining electromyographic (EMG) activity in 13 patients using surface electrodes for $S M$ and trapezius, and concentric needle electrodes for SC. At rest four patients had patterns of EMG activation which did not 
correspond to the predicted pattern. During voluntary rotation inappropriate (ipsilateral) activation of SM was frequently seen, and sometimes inappropriate contralateral SC activation, despite normal activation during other movements. Inappropriate inhibition of prime movers was observed in seven patients. In two of these SM activation was the inverse of normal. Botulinum toxin injections to prime movers failed to normalize patterns of muscle activation, despite clinical benefit. We conclude that torticollis is associated not only with over-activation of muscles as stressed in current theories of the pathogenesis of dystonia, but also with inhibition, as though motor programmes have been corrupted.
TOTAL LYMPHOID IRRADIATION IN MULTIPLE SCLEROSIS: A PRELIMINARY REPORT

CM Wiles, G Sawle, L Gilbert, T Ioannides. St Thomas' Hospital, London

Over two years 66 multiple sclerosis patients were referred for a trial of total lymphoid irradiation. Fifteen were unsuitable and 24 withdrew after receiving full information. Three patients were treated openly and 24 (mean age 37.5 years, 16 male) were randomised to receive either $1980 \mathrm{cGy}$ or sham treatment in 12 fractions each to the mantle and then to the inverted " $Y$ " field (including spleen) on a Philips SL75-10 8 MeV Linear Accelerator. Active treatment resulted in a fall in blood lymphocyte count from $2 \cdot 28$ $\left(\times 10^{9} / \mathrm{L}\right)$ pretreatment to 0.32 at end treatment, 0.78 at six months and 0.87 at one year. The expanded Kurtzke disability status score and Troiano scores significantly increased from $4.33+/-1.49$ and $2.59+/-1.22$ to $5.89+/-1.11$ and $4.63+/-1.98$ in the year prior to entry and continued to increase to $6.89+/-1.29$ and $6.36+/-2.44$ in 14 patients followed for one year. Walking speed declined for a year; video assessments were also used for ambulation assessment. One death occurred in a sham treated patient: six treated female patients developed amenorrhea and are on hormone replacement: one treated patient developed hepatitis due to baclofen and one a skin rash of uncertain origin. 